Notfall Rettungsmed 2019 $\cdot 22: 386-393$ https://doi.org/10.1007/s10049-018-0462-3 Online publiziert:29. Mai 2018

(C) Der/die Autor(en) 2018 CrossMark

\author{
N. Löber' · G. Kranz ${ }^{2}$ R. Berger' · A. Gratopp ${ }^{2}$ ·. S. Jürgensen ${ }^{3,4}$ \\ 'Klinisches Qualitäts- und Risikomanagement, Charité - Universitätsmedizin Berlin, Corporate Member of \\ Freie Universität Berlin, Humboldt-Universität zu Berlin, and Berlin Institute of Health, Berlin, \\ Deutschland \\ ${ }^{2}$ Klinik für Pädiatrie mit Schwerpunkt Pneumologie und Immunologie, Charité - Universitätsmedizin \\ Berlin, Corporate Member of Freie Universität Berlin, Humboldt-Universität zu Berlin, and Berlin Institute \\ of Health, Berlin, Deutschland \\ ${ }^{3}$ Charité - Universitätsmedizin Berlin, Corporate Member of Freie Universität Berlin, Humboldt-Universität \\ zu Berlin, and Berlin Institute of Health, EBC Hochschule, Berlin, Deutschland \\ ${ }^{4}$ Klinikum Stuttgart, Stuttgart, Deutschland
}

\title{
Inanspruchnahme einer pädiatrischen Notaufnahme
}

\section{Motivation und Besuchsgründe bei nichtdringlichem Behandlungsbedarf}

Facharztpatienten und nicht dringlichen Behandlungen zurückgeführt wird [1, $10,19]$.

Annahmen zu den patientenseitigen Motiven für die zunehmende Inanspruchnahme der akutmedizinischen Behandlungsangebote in Notaufnahmen werden intensiv diskutiert und untersucht. So konnten z. B. die subjektive Behandlungsdringlichkeit aus Sicht der Patienten bzw. Angehörigen [26, 27] und die hohe medizinische Qualität und Verfügbarkeit des Leistungsangebots als Besuchsmotive identifiziert werden [21, 23]. Auch die Nichtverfügbarkeit niedergelassener Ärzte trotz vorherigem Kontaktversuch während der regulären Praxisöffnungszeiten und die Unkenntnis weiterer Versorgungsangebote (wie z.B. der vertragsärztliche Bereitschaftsdienst) bewegen Patienten zu einem Besuch der Notaufnahmen in Krankenhäusern [28].

Für den Behandlungskontext der pädiatrischen Notfallversorgung in Krankenhäusern wurden bis dato in Deutschland kaum Studien durchgeführt, die die genauen Motive und Besuchsgründe von Eltern bzw. Angehörigen untersuchen. Ausländische Untersuchungen des pädiatrischen Notversorgungskontextes z. B. aus den USA $[9,29,30]$, Spanien [4, 11, 15], England [17], Österreich [13] oder Belgien [2] - lassen vermuten, dass die (vorab genannten) Gründe für eine Überfüllung von Notaufnahmen in der Erwachsenenmedizin auch ursächlich für die stärkere Inanspruchnahme pädiatrischer Notaufnahmen in Deutschland sein könnten. Ziel der vorliegenden Untersuchung ist es daher, durch Befragung Gründe und Motive für die Vorstellung von Kindern (durch ihre Eltern oder Angehörigen) mit nichtdringlichem Behandlungsbedarf in einer pädiatrischen Notaufnahme zu erfahren.

\section{Methodik und Stichproben- bildung}

Eltern und andere Angehörige, die mit Kindern (als Patienten) die pädiatrische Notaufnahme eines im westlichen Bereichs der Berliner Innenstadt liegenden Klinikums der Maximalversorgung aufsuchten sowie minderjährige Patienten ohne Begleitung wurden dafür über einen Zeitraum von 4 Wochen mit Hilfe eines Fragebogens zu den Gründen ihrer Vorstellung in der pädiatrischen Notaufnahme standardisiert befragt.

Für die Befragung wurde ein bestehendes Instrument aus einer anderen Studie [28] in einem mehrstufigen, interdisziplinären Abstimmungsprozess (unter Einbeziehung von Pädiatern, Gesundheits- und Kinderkrankenpflegern sowie Epidemiologen) auf den spezifi- 


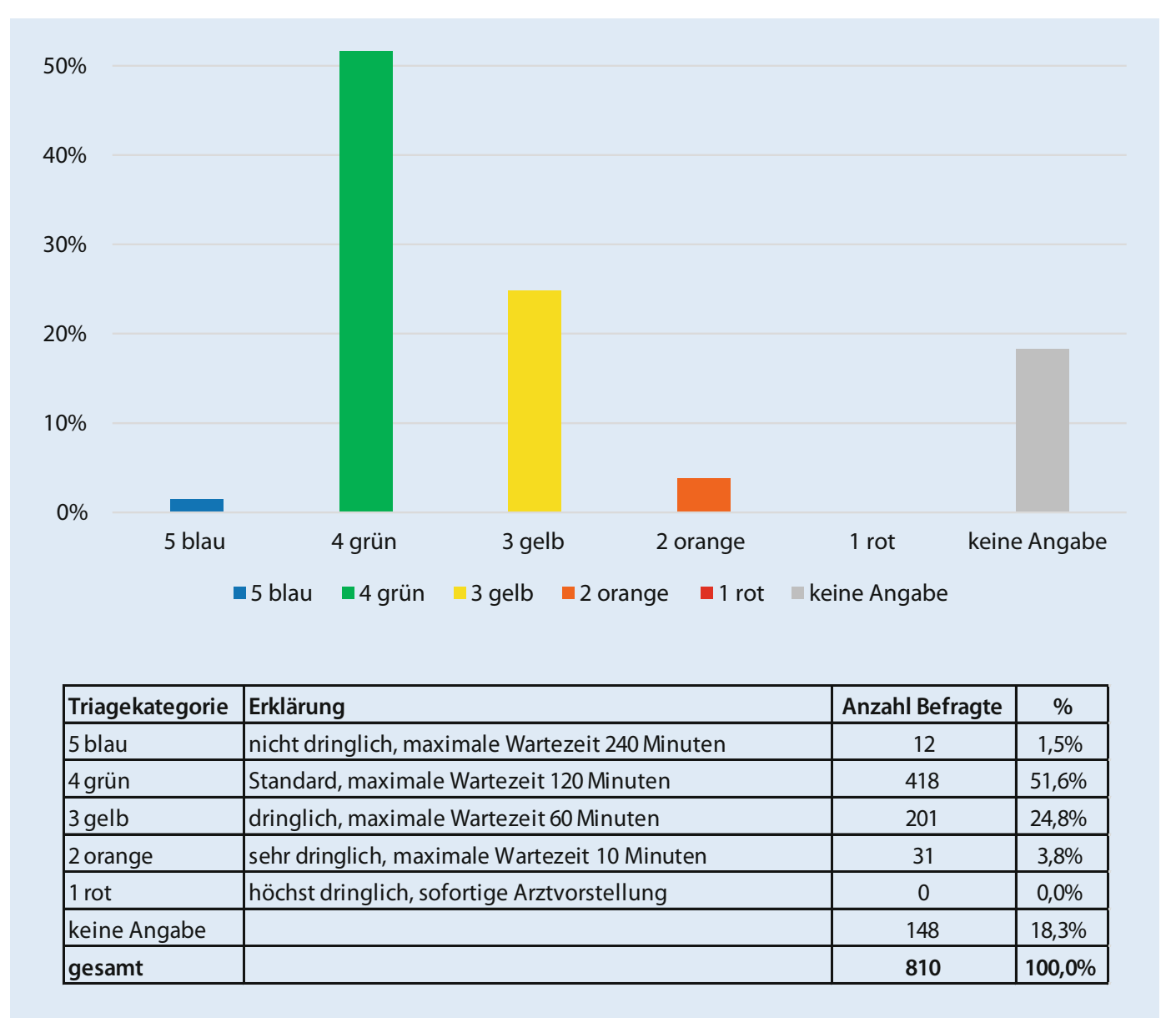

Abb. $1 \triangleleft$ Manchester Triage Verteilung der untersuchten Stichprobe $(n=810)$ schen Befragungskontext angepasst. Zur Wahrung der Anonymität wurde auf persönliche Angaben verzichtet, sodass keine Rückschlüsse auf einzelne Patienten möglich waren. Um der zunehmenden Anzahl nicht deutschsprachiger Eltern und Patienten gerecht zu werden, wurde das entwickelte Befragungsinstrument von Muttersprachlern aus dem eigenen Haus in 5 Sprachen übersetzt (Englisch, Serbisch-Kroatisch, Russisch, Türkisch und Farsi) und zur psychometrischen Validierung rückübersetzt.

Der Fragebogen wurde mit einem Informationsblatt im Rahmen der administrativen Aufnahme nach der Triagierung ausgehändigt und während der Wartezeit (d.h. vor der eigentlichen Behandlung durch einen Arzt) von den Probanden ausgefüllt. Das Informations- bzw. Erläuterungsblatt der Studie umfasste Angaben zum Befragungshintergrund, zu Datenschutz- und Genderaspekten sowie eine postalische, elektronische und telefonische Kontaktmöglichkeit für Rückfragen. Es wurden sowohl Patienten/Angehörige berücksichtigt, die per Krankentransport oder Notfallrettung eingeliefert worden waren, als auch Patienten/Angehörige, die eigenständig die pädiatrische Notaufnahme aufgesucht hatten. Zur Eingrenzung der Patientengruppe mit nichtdringlichem Behandlungsbedarf wurde das in der Notaufnahme etablierte „Manchester triage system" (MTS) angewendet, mit Hilfe dessen Pflegende die Behandlungsdringlichkeit anhand von Beschwerdebildern und Vitalfunktionen zuordnen können [20, 31]. Das MTS umfasst 5 Stufen, denen maximale Systemzielzeiten bis zum Arztkontakt zugeordnet sind:

- Rot (1): höchst dringlich, sofortige Arztvorstellung,

- Orange (2): sehr dringlich, maximale Wartezeit $10 \mathrm{~min}$,

- Gelb (3): dringlich, maximale Wartezeit $60 \mathrm{~min}$,

- Grün (4): Standard, maximale Wartezeit $120 \mathrm{~min}$,
- Blau (5): nicht dringlich, maximale Wartezeit $240 \mathrm{~min}$ [16].

In einem ersten Schritt wurden Patienten mit MTS-Kategorie 1 von der Befragung ausgeschlossen, da diese definitionsgemäß höchstdringliche Notfälle sind und ohne Wartezeit (und ohne Möglichkeit zum Ausfüllen des Fragebogens) sofort dem behandelnden Arzt vorgestellt werden (müssen). Bei der verbliebenen Patientengruppe wurde in einem zweiten Schritt die Sprachfähigkeit des Angehörigen bzw. Patienten geprüft und auf eine Ausgabe des Fragebogens verzichtet, wenn eine Verständigung über die deutsche oder eine der 5 fremdsprachlichen Versionen nicht möglich war. Die subjektive Behandlungsdringlichkeit wurde durch die Parameter „empfundene Schmerzen“ und „bestehende Dauer der Beschwerden“ erfasst. Die allgemeine Volatilität des Patientenaufkommens in der Notaufnahme führte schließlich dazu, dass in Zeiten 
mit besonders hohem Besucheraufkommen auf die Ausgabe des Fragebogens zugunsten der schnelleren administrativen Aufnahme verzichtet wurde. Um zu überprüfen, ob sich die befragte Gruppe (Stichprobe) von der Gesamtheit der im betrachteten Zeitraum behandelten Patienten $(n=2288)$ unterscheidet, wurde die Altersverteilung beider Kollektive mithilfe eines $\chi^{2}$-Anpassungstests verglichen. Im Ergebnis zeigte sich, dass sich die befragte Gruppe von der Gesamtheit nicht signifikant unterscheidet $(p=0,387)$.

Postleitzahl, Fachdisziplin (Pädiatrie und Kinderchirurgie) sowie die Triagekategorie wurden vor Ausgabe der Fragebögen an die Probanden (wenn möglich durch die Pflegekräfte) auf dem Bogen dokumentiert; ausgefüllte Fragebögen konnten über einen Briefkasten an der administrativen Aufnahme zurückgegeben werden. Für die Datenauswertung wurden kategoriale Fragen deskriptiv-statistisch mit Hilfe von IBM SPSS (Version 24; Armonk, NY, USA)) verarbeitet und handschriftliche Freitextkommentare in eine Datenbank transkribiert und kategorisiert.

\section{Ergebnisse}

Insgesamt verzeichnete die pädiatrische Notaufnahme im 4-wöchigen Betrachtungszeitraum 1881 Patientenkontakte (bzw. Patientenbesuche mit Eltern oder Angehörigen) in den MTS-Kategorien 2-5, von denen 810 mit den verschiedenen Sprachversionen des Fragebogens befragt werden konnten. Dies entspricht - bezogen auf die Grundgesamtheit einer Stichprobengröße von $43 \%$ für die hier vorgestellte Untersuchung. Gut 2/3 der beantworteten Fragebögen wurden der konservativen Behandlungseinheit der Notaufnahme zugeordnet, die übrigen Fälle kinderchirurgisch versorgt. Über $90 \%$ der Kinder wurden von einem Elternteil, d.h. von der Mutter $(67,1 \%)$ oder dem Vater $(24,3 \%)$, in der Notaufnahme vorgestellt; die übrigen Kinder wurden von nahen Verwandten und Betreuern (z.B. Lehrer, Erzieher) in die Notaufnahme gebracht oder stellten sich dort selbst vor. Ausgewertet wurden Fragebögen, bei denen min-

Notfall Rettungsmed 2019 - 22:386-393 https://doi.org/10.1007/s10049-018-0462-3

(c) Der/die Autor(en) 2018

N. Löber · G. Kranz · R. Berger · A. Gratopp · J. S. Jürgensen

Inanspruchnahme einer pädiatrischen Notaufnahme. Motivation
und Besuchsgründe bei nichtdringlichem Behandlungsbedarf

\section{Zusammenfassung}

Hintergrund. Die Zahl der Patienten in den pädiatrischen Notaufnahmen der Krankenhäuser steigt an. Die patientenseitigen Beweggründe für den Besuch von Notaufnahmen werden zwar für den Erwachsenenbereich zunehmend untersucht und sind bekannt, nicht aber für den speziellen Fall der Kindernotfallversorgung. Ziel der Arbeit. Ziel der Studie war, Gründe und Motive für die Vorstellung von Kindern (durch ihre Eltern oder Angehörigen) mit nichtdringlichem Behandlungsbedarf in einer pädiatrischen Notaufnahme zu erfassen. Methodik. Befragung von Patienten bzw. deren Angehörigen in einer großstädtischen pädiatrischen Notaufnahme mit Hilfe eines standardisierten Fragebogens über einen Zeitraum von 4 Wochen.

Ergebnisse. Insgesamt konnten 810 Probanden befragt werden. Die zentralen Erkenntnisse decken sich mit internationalen Studienergebnissen, insbesondere aber mit jüngeren deutschen Studien zu vergleichbaren Fragestellungen. Motivgruppen für das
Aufsuchen der Notaufnahme aus Eltern- bzw. Patientensicht sind: individuelle Präferenzen der Patienten, vornehmlich geografische Nähe und erwartetes Versorgungsspektrum; fehlende Verfügbarkeit/Erreichbarkeit ambulanter Versorgungsmöglichkeiten; mangelnde Kenntnis alternativer ambulanter Notfallstrukturen. Bei mehr als die Hälfte der Patienten handelt es sich nach objektiver Dringlichkeitseinschätzung um keine Notfallpatienten, die folglich keiner Behandlung in einer pädiatrischen Notaufnahme bedürft hätten.

Diskussion. Eine verbesserte Steuerung der Patientenströme und eine Reformierung der intersektoralen, pädiatrischen Notfallversorgung scheinen in Anbetracht der anhaltend hohen Patientenströme in (pädiatrischen) Notaufnahmen angebracht.

\section{Schlüsselwörter}

Kindernotfallmedizin · Notfallmedizin . Notfallbehandlung · Notfallversorgung . Intersektorale Zusammenarbeit

\section{Use of a pediatric emergency department. Reasons for nonurgent patient visits}

\section{Abstract}

Background. The number of patients treated at German pediatric emergency departments is rising. While considerate research has been undertaken to assess reasons for adult emergency department visits, little is known about the specific case of pediatric emergency provision.

Objective. The aim of this study was to evaluate motivations for nonurgent patient visits in the pediatric emergency department. Materials and methods. Standardized survey of patients (or their respective relatives) seeking pediatric emergency care in a metropolitan pediatric emergency department over a 4-week period.

Results. A total of 810 subjects were included in the study. The central findings support the results of comparable international studies and simultaneously match contemporary German studies. From a pediatric/parent perspective, the most common motives for visiting an emergency department are the following: individual preferences, mostly geographical proximity and expected spectrum of care; limited accessibility/reachability of other existing ambulatory care structures; insufficient knowledge regarding alternative ambulant emergency care structures.

Conclusion. Over $50 \%$ of children using the services of pediatric emergency departments can be classified as nonurgent cases that do not require emergency treatment. As pediatric emergency departments in Germany remain in high demand, more efficient steering of patient flow and a reform of intersectoral, pediatric emergency care are necessary.

\section{Keywords}

Pediatric emergency medicine - Emergency medicine - Emergency treatment . Intersectoral collaboration - Intersectoral cooperation 


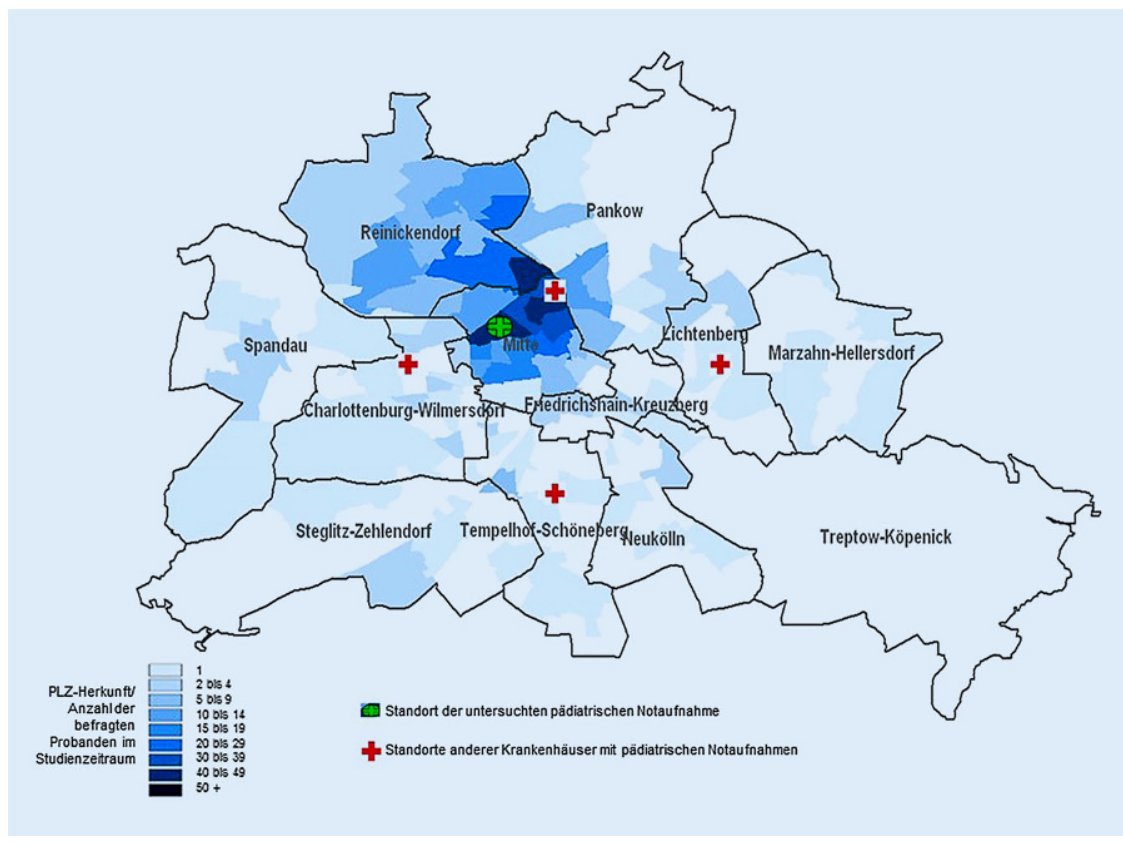

Abb. 2 A Geografische Herkunft der befragten Probanden/Distanz zur untersuchten pädiatrischen Notaufnahme $(n=729)$

destens eine Frage beantwortet worden war. Dies führte in der Konsequenz zu unterschiedlichen Rückläufen je Frage.

\section{Medizinische und subjektive Dringlichkeit}

Die Klassifikation und Priorisierung der medizinischen Behandlungsdringlichkeit von Kindern in der pädiatrischen Notaufnahme erfolgt nach MTS (• Abb. 1).

Über die Hälfte der vorgestellten Kinder hatten mit blauer oder grüner MTS-Klassifizierung normalen Behandlungsbedarf und sind keine klassischen Notfallpatienten mit hoher Behandlungsdringlichkeit. Einen dringenden Behandlungsbedarf (gelb) wiesen 24,8\% der Kinder auf; 3,8\% sogar einen sehr dringenden Behandlungsbedarf (orange). Bei gut $18 \%$ der Probanden wurde die Triagekategorie im Rahmen der Fragebogenausgabe nicht vermerkt.

Im Befragungszeitraum ambulant behandelt wurden $85,1 \%$ aller Patienten; $14,9 \%$ aller vorgestellten Kinder wurden in Folge der Erstbehandlung in der Notaufnahme stationär im Haus zur weiteren Behandlung aufgenommen. Über die Hälfte der befragten Eltern bzw. Patienten $(57,2 \%)$ gaben an, dass die ge- sundheitlichen Beschwerden, die sie zum Aufsuchen der Notaufnahme bewegt hatten, weniger als $24 \mathrm{~h}$ bestanden hatten. Bei $17,4 \%$ der Patienten lagen die Beschwerden bereits seit bis zu 2 Tagen vor, noch 13,9\% der Befragten gaben an, dass die körperlichen Beschwerden sogar länger als 2 und bis zu 7 Tagen bestanden hatten. Länger als 1 Woche oder gar länger als 1 Monat hatten Beschwerden immerhin noch bei 7,2\% bzw. 4,3\% der befragten Besucher bestanden. Schmerzen auf einer nummerischen Analogskala von 0-10 dokumentierten 645 von 810 befragten Eltern bzw. Patienten (79,9\%), wobei $17,8 \%$ der Befragten die eigenen Schmerzen oder die ihrer Kinder als stark beurteilten (Ausprägungen von 8-10).

\section{Geografische Einzugsweite der Notaufnahme}

Anhand der bei 729 von 810 Probanden erfassten Postleitzahlen (PLZ) konnte eine durchschnittliche geografische Distanz zwischen Wohnort und der pädiatrischen Notaufnahme berechnet werden, die das Einzugsgebiet widerspiegelt. Über die Hälfte der Eltern bzw. Kinder (55,7\%) besuchten die Einrichtung aus einem Einzugsradius von maximal $5 \mathrm{~km}$ Entfernung. Gleichzei- tig gaben 35,3\% der Befragten an, die Notaufnahme aufgrund der örtlichen Nähe (zum Wohnort) aufgesucht zu haben. Ein knappes Viertel $(24,8 \%)$ der Besucher reiste bereits aus bis zu $10 \mathrm{~km}$ Entfernung zur Notaufnahme an.

Eine Analyse der PLZ zeigt, dass die untersuchte Notaufnahme schwerpunktmäßig von Eltern und Patienten aus dem Nordwesten Berlins besucht wird (• Abb. 2).

Neben der untersuchten Notaufnahme sind in $\bullet$ Abb. 2 zusätzlich kinderärztliche Erste-Hilfe-Stellen in Kooperationskrankenhäusern der KV (Kassenärztliche Vereinigung) sowie eine von der KV organisierte Erste-Hilfe-Stelle verzeichnet (Kreuze). Dunkle Flächen markieren die besucherstarken PLZ-Bereiche, hellere Flächen PLZ-Bereiche, aus denen weniger Patienten innerhalb des Befragungszeitraums zur untersuchten Notaufnahme anreisten.

\section{Vorkontakt mit ambulanten Versorgungsstrukturen}

Vor dem Besuch der pädiatrischen Notaufnahme hatten 49,6\% (339/684) der befragten Besucher versucht, einen niedergelassenen Hausarzt oder Kinderarzt zu kontaktieren. Dabei gaben 20,2\% der Befragten an, vom Arzt direkt in die Notaufnahme verwiesen worden zu sein, bei $22,7 \%$ der Befragten blieb der Kontaktversuch mit dem niedergelassenen Arzt aufgrund geschlossener Praxen (Urlaub oder keine Sprechzeiten) erfolglos. Dies antizipierten bereits 10,4\% der Befragten, die angaben, den Hausarzt aufgrund vermutlich nicht kurzfristig verfügbarer Termine gar nicht erst kontaktiert zu haben. Das Auftreten der Notfallsituation außerhalb von Sprechzeiten des niedergelassenen Arztes (z. B. an Wochenenden) oder die subjektiv gefühlt hohe medizinische Dringlichkeit waren weitere explizit genannte Gründe, den Hausarzt oder Kinderarzt vor dem Besuch in der Notaufnahme nicht zu kontaktieren. Eine Übersicht zu den Kontakt- bzw. Nichtkontaktgründen zeigt $\bullet$ Abb. 3 .

Der Fragebogen wurde von 46,2 \% der Probanden an Tagen und zu Zeiten ausgefüllt, in denen Haus- und Kinderärzte typischerweise Sprechstunden anbieten 


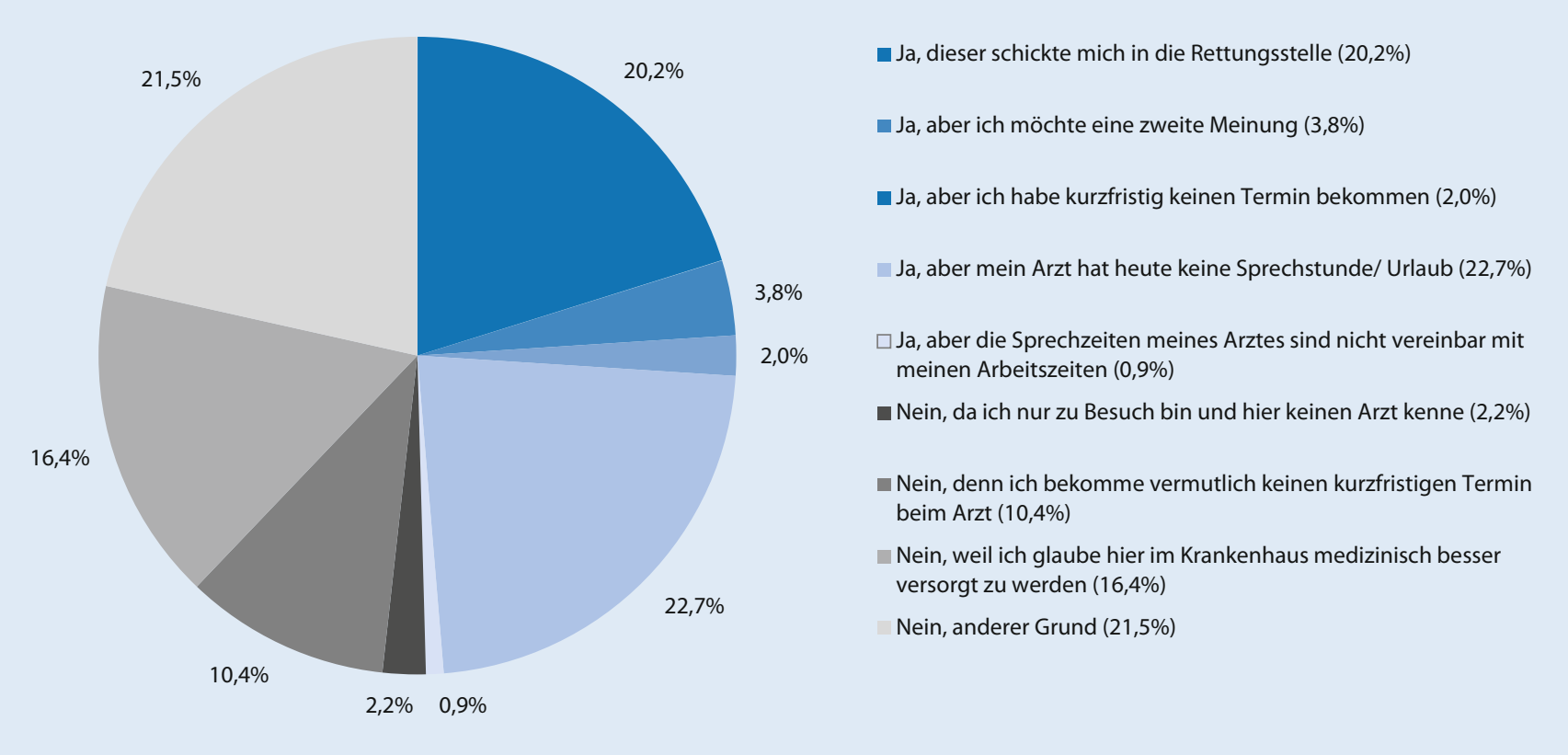

Abb. 3 ॥ Antworten auf die Frage „Haben Sie zuvor einen niedergelassenen Arzt (Hausarzt/Kinderarzt) wegen Ihrer Beschwerden/wegen der Beschwerden Ihres Kindes kontaktiert?" $(n=684)$

(Montag bis Freitag von 8-19 Uhr exklusive Mittwochnachmittag).

\section{Kenntnis alternativer Versorgungs- strukturen}

Insbesondere außerhalb der regulären Sprechstundenzeiten der niedergelassenen Ärzte sind bei Eltern Kenntnisse alternativer Versorgungsstrukturen erforderlich, um die eingangs geschilderten Herausforderungen des „crowding“ mit Nichtnotfallpatienten in der Notaufnahme zu reduzieren. Nur gut $1 / 3$ der Probanden kennt alternative Versorgungsangebote, wobei lokale Notfalleinrichtungen deutlich bekannter sind als der bundesweite telefonische Notdienst/ Bereitschaftsdienst der KV, den nur 7,4\% der Befragten kennen (• Abb. 4).

Von den Eltern, die den telefonischen KV-Notdienst kennen und auch tatsächlich angerufen hatten, wurden $73 \%$ direkt und ohne Untersuchung, die übrigen $27 \%$ erst nach körperlicher Untersuchung durch den KV-Notdienst in die Kindernotaufnahme verwiesen. Offensichtlich hatten jedoch viele Eltern trotz Kenntnis des telefonischen Bereitschaftsdienstes diesen nicht angerufen. Die Beweggründe hierfür wurden als offene Frage erhoben und umfassen u. a.:
- Bequemlichkeitsmotive („Weil ich neben dem Krankenhaus wohne“),

- Vorerfahrungen („Uns wurde bereits mehrfach gesagt, dass dort keine Kinderärzte tätig seien“; „Schlechte Erfahrung, Person am anderen Ende schien nicht qualifiziert, außerdem erschien uns eine Ferndiagnose nicht sinnvoll“; „Sind unhöflich und sagen man soll in die Erste Hilfe fahren“; „Damals einmal angerufen als er ein kleines Baby war, da hieß es, wegen so was bitte in die Rettungsstelle fahren"),

- fehlende Diagnosemöglichkeiten („Kind ist gestürzt mit Verdacht auf Gehirnerschütterung, die Untersuchungsmöglichkeiten bei Ihnen sind größer“; „Weil dieser [KV-Notdienst] kein Sono und keine Blutuntersuchung machen kann“; „Weil im Krankenhaus mehr Möglichkeiten bestehen") bis hin $\mathrm{zu}$,

- lange Wartezeiten („Es geht schneller in der Rettungsstelle als auf einen KV-Notdienst zu warten“; „Weil der KV-Arzt meistens mindestens drei Stunden braucht, um zu kommen, und im Krankenhaus sind wir besser aufgehoben").

\section{Spezifische Besuchsbeweggründe}

Obwohl gut 1/3 der befragten Probanden alternative Versorgungsstrukturen kannte, erfolgte die bewusste Entscheidung zum Aufsuchen der hier untersuchten pädiatrischen Notaufnahme (• Abb. 5).

Über 1/3 der Eltern bzw. Begleitpersonen suchte die Notaufnahme aufgrund der räumlichen Nähe auf; dies deckt sich mit den eingangs analysierten PLZ-Einzugsgebieten. Weitere $32 \%$ der Befragten besuchten die Notaufnahme aufgrund guter vorheriger Vorerfahrungen. Auch gaben kumuliert $49 \%$ der Befragten auf die Frage „Sind Sie in den letzten 12 Monaten mit ihrem Kind bereits in einer (Kinder-)Rettungsstelle gewesen?" $(n=732)$ an, eine solche einmal oder sogar mehrmals im vergangenen Jahr aufgesucht zu haben. Da die untersuchte Notaufnahme einer hochspezialisierten pädiatrischen Maximalversorgungsstruktur (mit zahlreichen Fachambulanzen, sozialpädagogischem Zentrum usw.) angehört, nutzten sie etwa $10 \%$ der Probanden als schnellen Zugangsweg zur weiteren Dauerbehandlung. Hierbei handelt es sich vermutlich vornehmlich um chronisch kranke Kinder mit berechtigter Vorstellung in einem Haus der Maximalversorgung. 


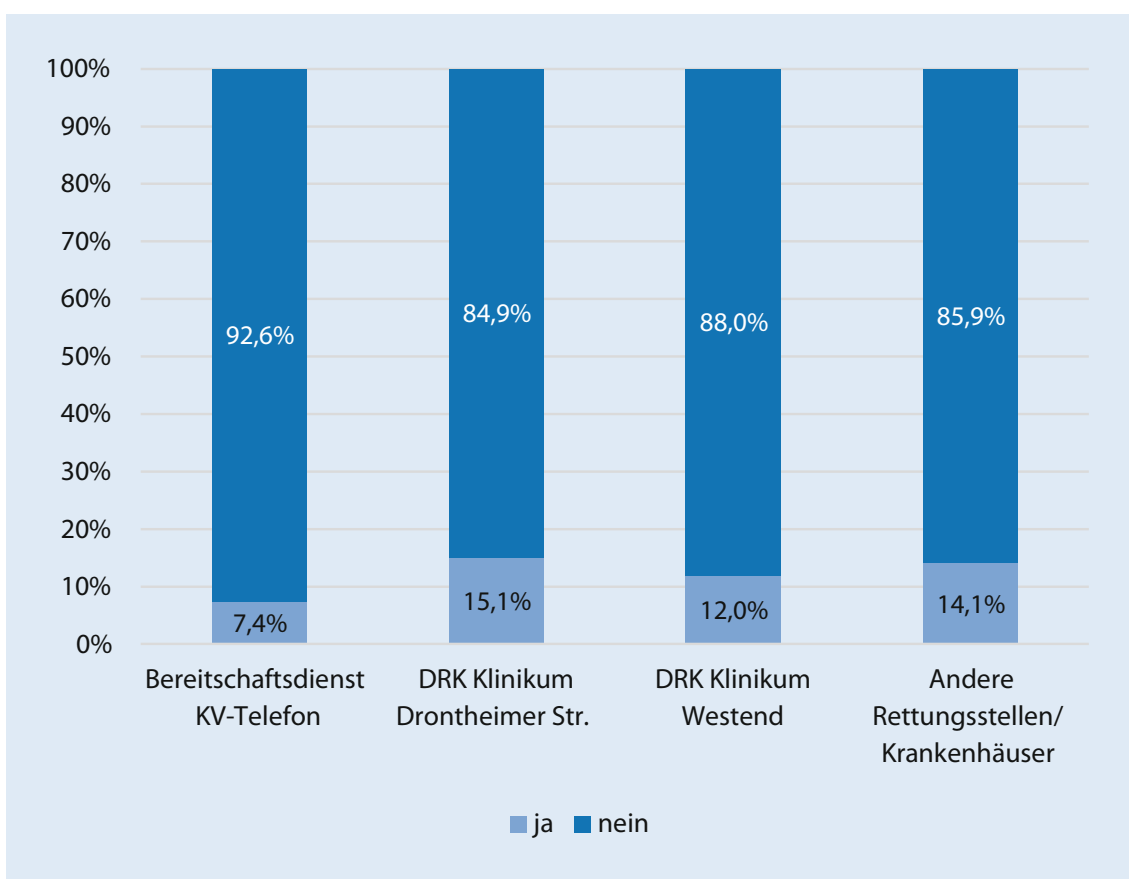

Abb. $4 \Delta$ Kenntnis alternativer Versorgungsstrukturen $(n=810)$

\section{Diskussion}

\section{Individuelle Präferenzen}

Eltern suchen eine Notaufnahme häufig aufgrund von Verunsicherung und Besorgnis auf [13]. Die Angst, dass eine vermutlich schwerwiegende Erkrankung oder Beschwerde des eigenen Kindes vorliegen könnte, führt naturgemäß zum Aufsuchen einer geografisch nahen, immer besetzten und zudem medizinisch gut ausgestatteten Behandlungseinrichtung. Dies deckt sich mit anderen Untersuchungen, die z. B. die subjektive Notfalleinschätzung/ Behandlungsdringlichkeit von erwachsenen Patienten $[26,27]$ und die hohe medizinische Qualität und Verfügbarkeit des Leistungsangebots als Besuchsmotive identifizieren konnten. Erkennbar sind hier bequemlichkeitsorientierte Präferenzmuster, die in einer anderen Studie [23] treffend mit den Anglizismen „doc to go", „high-tech statt Hausarzt" und „all inclusive“ umschrieben werden und nicht zwangsläufig mit dem objektiven (Nicht-)Dringlichkeitsgrad der wahrgenommenen Beschwerden übereinstimmen. Da fast die Hälfte der Eltern (49\%) bereits zum zweiten Mal binnen 12 Monaten in einer Notaufnahme vor- stellig wurde, wird die hier untersuchte Notaufnahme vermutlich von einigen Patienten als bequemer Hausarztersatz verstanden und genutzt.

\section{Fehlende Verfügbarkeit/ \\ Erreichbarkeit ambulanter Versorgungsmöglichkeiten}

Diese Präferenzen bzw. Anspruchshaltungen treffen auf eine lokal suboptimal ausgestaltete niedergelassene Versorgungsstruktur, trotz eines nominellen ambulanten pädiatrischen Versorgungsgrads von 147,9\% aus Sicht der KV [12]: Fast 1/4 aller Befragten konnte den niedergelassenen Arzt aufgrund geschlossener Praxen nicht erreichen. Dies deckt sich mit Erkenntnissen anderer Untersuchungen $\mathrm{zu}$ erwachsenen Patienten, die nichtgeöffnete (Hausarzt-)Praxen als manifeste Barriere für die Nutzung niedergelassener Versorgungsstrukturen erkannten $[3,8,21]$. Zwar erklären Nachtzeiten und Wochenenden mit ohnehin reduzierter Versorgungskapazität dies partiell, viele der in diesen Zeiten in der Notaufnahme vorstelligen Kinder bedürfen jedoch keiner Notfallversorgung in einem Krankenhaus der Maximalversorgung und verstärken so möglicherweise den eingangs erläuter- ten Effekt des „crowding“. Verglichen mit der Ersteinschätzung vor Ort und der nach der Behandlung resultierenden stationären Aufnahmequote von lediglich $14,9 \%$ scheinen die niedergelassenen Haus- bzw. Kinderärzte ihrer Lotsenfunktion im Gesundheitswesen nur bedingt gerecht zu werden. Ebenfalls könnte eine möglicherweise nicht stringent genug umgesetzte Vertretungspflicht in den Praxen mitursächlich für die fehlende Erreichbarkeit niedergelassener (Kinder-) Ärzte sein.

\section{Fehlende Kenntnis alternativer Notfallversorgungsstrukturen}

Unabhängig von der tatsächlichen Behandlungsnotwendigkeit in einer Notfallversorgungseinrichtung sind die etablierten Versorgungsstrukturen des ambulanten, kassenärztlichen Versorgungsbereichs wenig bekannt; auch lokale, vertraglich gesteuerte KV-Kooperationseinrichtungen sind den meisten Eltern nicht geläufig. Dies könnte - in Kombination mit den teils frustranen Kontaktversuchen zum niedergelassenen Arzt - mitverantwortlich für eine erhöhte Vorstellung nichtkritischer Patienten in der Notaufnahme sein.

\section{Limitationen}

Trotz Ergebnisanalogie zu anderen Untersuchungen ist die durchgeführte Studie mit Limitationen verbunden und in ihrer Aussagekraft nicht zwingend generalisierbar. Die gravierendste Einschränkung liegt im Befragungsszenario der Studie begründet: Mit wenigen Ausnahmen wurden nicht die minderjährigen Patienten selbst, sondern deren Begleiter befragt, was zu einem Informationsbias (insbesondere in Bezug auf subjektive Parameter wie empfundene Schmerzen und Dauer der Beschwerden) führen kann. Insgesamt beantworteten $43 \%$ der Besucher den Fragebogen im Untersuchungszeitraum; Patienten mit sofortigem bzw. sehr dringendem Behandlungsbedarf waren a priori von der Studie ausgeschlossen. Die Antworten der nicht teilnehmenden Patienten/ Angehörigen hätten mitunter anders ausfallen können, was die Gefahr ei- 


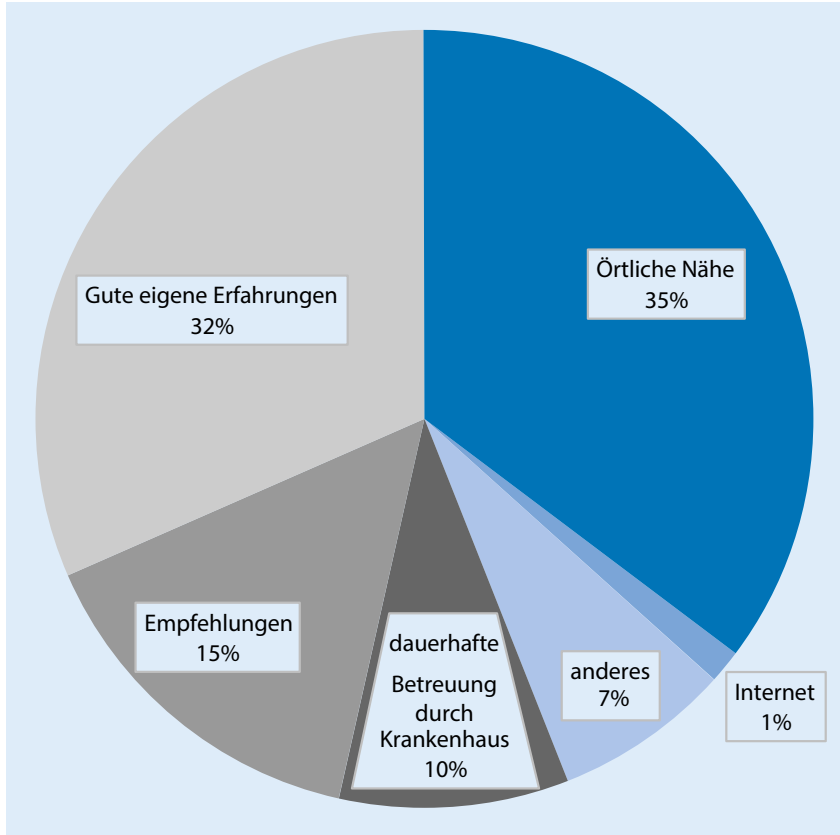

Abb. $5<$ Antworten auf die Frage „Was hat Sie bewogen, gerade unsere Kinderrettungsstelle aufzusuchen?" $(n=504)$

nes Selektionsbias in sich trägt. Auch das spezifische, nominell überversorgte pädiatrische Versorgungsnetz in einem großstädtischen Ballungszentrum ist nicht repräsentativ für die deutsche $\mathrm{Ge}$ sundheitsversorgungslandschaft; in regionalen (z. B. ländlichen) Gebieten mit Krankenhäusern anderer Versorgungsstufen könnten die Präferenzen und Motivationen von Eltern bzw. Patienten unterschiedlich ausgeprägt sein. Auch die Wahl anderer Befragungszeiträume könnte zu divergenten Ergebnissen führen. Schlussendlich führt der Einschluss von via Rettungswagen eingelieferten $\mathrm{Pa}$ tienten (in den MTS-Kategorien orange bis blau) zu einem leichten Vergleichsbias in Bezug zu anderen Studien, die Rettungsdienstpatienten a priori ausgeschlossen hatten. Dieser Bias umfasst aber maximal das Teilkollektiv orange triagierter Patienten $(n=31 ; 3,8 \%$ aller befragten Probanden).

\section{Fazit für die Praxis}

Im Ergebnis zeigt die durchgeführte Studie zur Inanspruchnahme einer pädiatrischen Notaufnahme trotz unterschiedlicher Methodik und der damit verbundenen beschränkten Vergleichbarkeit Ergebnisparallelen zu anderen Studien im Erwachsenenkontext [21, 23, 28]:
- Die beobachtete unangebrachte Inanspruchnahme, teils aufgrund eigener, nutzenoptimierender Präferenzen, wird durch die restriktionsfreie Arztwahl begünstigt und nicht durch entsprechend effektive Steuerungsmechanismen ambulanter Notfalleinrichtungen durch die KV verringert.

- Weder die personelle noch die finanzielle Ausstattung der untersuchten pädiatrischen Notaufnahme sind auf die zunehmenden, nichtdringlichen Patientenströme adäquat vorbereitet; Erwartungshaltungen von Eltern und Patienten (z. B. in Bezug auf Wartezeiten in der Notaufnahme) werden so in zunehmendem Maße enttäuscht.

- Einerseits bedarf es deshalb einer konsequenteren Wahrnehmung des Versorgungsauftrags niedergelassener (Kinder-)Ärzte, andererseits neuer und adäquater (Notfall-)Versorgungsstrukturen und Steuerungsmöglichkeiten für solche Patienten, die absehbar keiner stationären Weiterbehandlung im Krankenhaus bedürfen.

- Die derzeit in Deutschland intensiv diskutierten und teilweise bereits eingeführten Instrumente wie z. B. die Eröffnung von Portalpraxen in Krankenhäusern [6] oder der Aufbau in- tegrierter Leitstellen/Notfallzentren [22], weisen in die richtige Richtung. Kürzlich vorgelegte Modelle des Sachverständigenrates für Gesundheit, des Spitzenverbandes der gesetzlichen Krankenkassen und der Allgemeinen Ortskrankenkassen zur verbesserten Zusammenarbeit von niedergelassenen Ärzten und Klinikärzten im Rahmen der ambulanten Notfallversorgung unterscheiden sich insbesondere durch den unterschiedlich aufgefassten Sicherstellungsauftrag.

- Das für das Frühjahr 2018 vom Sachverständigenrat angekündigte Gutachten zur Notfallversorgung [5] wird die kontrovers geführte Debatte sicherlich weiter fokussieren und hoffentlich dazu führen, dass die starren Sektorengrenzen zum Wohle patientenorientierter Notfallversorgungsstrukturen aufgebrochen und neu sortiert werden [7].

\section{Korrespondenzadresse}

\section{N. Löber}

Klinisches Qualitäts- und Risikomanagement, Charité - Universitätsmedizin Berlin, Corporate Member of Freie Universität Berlin, HumboldtUniversität zu Berlin, and Berlin Institute of Health

Charitéplatz 1, 10117 Berlin, Deutschland nils.loeber@charite.de

\section{Einhaltung ethischer Richtlinien}

Interessenkonflikt. N. Löber, G. Kranz, R. Berger, A. Gratopp und J.S. Jürgensen geben an, dass kein Interessenkonflikt besteht.

Dieser Beitrag beinhaltet keine von den Autoren durchgeführten Studien an Menschen oder Tieren.

Open Access. Dieser Artikel wird unter der Creative Commons Namensnennung 4.0 International Lizenz (http://creativecommons.org/licenses/by/4.0/deed. de) veröffentlicht, welche die Nutzung, Vervielfältigung, Bearbeitung, Verbreitung und Wiedergabe in jeglichem Medium und Format erlaubt, sofern Sie den/die ursprünglichen Autor(en) und die Quelle ordnungsgemäßnennen, einen Linkzur Creative Commons Lizenz beifügen und angeben, ob Änderungen vorgenommen wurden. 


\section{Literatur}

1. Arrubla P, Arias Ruiz S, Carrasquilla Villa G et a (2009) Demandas inadecuadas en urgencias e identificación del uso inadecuado de la hospitalización en el centro piloto de Assbasalud Ese. en Manizales. Año 2008. Arch Med 9:25-34

2. Benahmed N, Laokri S, Zhang WH et al (2012) Determinants of nonurgent use of the emergency department for pediatric patients in 12 hospitals in Belgium. Eur J Pediatr 171:1829-1837

3. Durand A-C, Palazzolo S, Tanti-Hardouin N et al (2012) Nonurgent patients in emergency departments: rational or irresponsible consumers? Perceptions of professionals and patients. BMC Res Notes 5:525

4. Fernandez Cano G, Martin Carballo G (2000) Urgencias pediatricas atendidas en una consulta de atencion primaria (I): Analisis de la demanda. Aten Primaria 26:76-80

5. Fricke A (2017) Gesundheitsweise wollen die Notfallversorgung aufmischen. ÄrzteZeitung 08.09.2017. https://www. aerztezeitung.de/politik_gesellschaft/notfallu-katastrophen-versorgung/article/942665/ praxen-laenger-oeffnen-gesundheitsweisewollen-notfallversorgung-aufmischen.html. Zugegriffen: 17. Nov. 2017

6. Fricke A (2017) Triage: Ärzte fordern Auflösen der Interessenkonflikte. ÄrzteZeitung 08.09.2017. https://www.aerztezeitung.de/ politik gesellschaft/berufspolitik/article/ 942680/triage-aerzte-fordern-aufloeseninteressenkonflikte.html. Zugegriffen: 17. Nov. 2017

7. Fricke $A$ (2017) Ab in die Klinik bei leichten Blessuren? Patientensteuerung sorgt für kontroverse Debatte. ÄrzteZeitung 24.10.2017. https:// www.aerztezeitung.de/politik gesellschaft/ versorgungsforschung/article/946133/ab-klinikleichten-blessuren-patientensteuerung-sorgtkontroverse-debatte.html?sh=1\&h=749753494. Zugegriffen: 17. Nov. 2017

8. Gill JM, Riley AW (1996) Nonurgent use of hospital emergency departments: urgency from the patient's perspective. J Fam Pract 42:491-496

9. Halfon N, Newacheck PW, Wood DL et al (1996) Routineemergency department usefor sick care by children in the United States. Pediatrics 98:28-34

10. Howard MS, Davis BA, Anderson C et al (2005) Patients' perspective on choosing the emergency department for nonurgent medical care: a qualitative study exploring one reason for overcrowding. JEmerg Nurs 31:429-435

11. Jimenez Alvarez $C$, Alaminos Mingorance $M$, Ruiz Montes AM et al (2000) Demanda urgente en cirugia infantil. Estudio de utilizacion inapropiada. CirPediatr 13(2):64-68

12. Kassenärztliche Vereinigung Berlin (2015) Bedarfsplan 2013 für den Zulassungsbezirk Berlin (ergänzt mit Wirkung vom 30.09.2015) https://www.kvberlin.de/20praxis/10zulassung/ 55bedarfsplan/bedarfsplan2013_erg.pdf. Zugegriffen: 11.Dez. 2017

13. Koller D, Damm L (2013) Behandlung akut erkrankter Kinder und Jugendlicher. Padiatr Padol 48:61-65

14. Köster C, Wrede S, Herrmann T et al (2016) Ambulante Notfallversorgung, AQUAInstitut. Göttingen. https://www.aquainstitut.de/fileadmin/aqua_de/Projekte/335 Ambulante Notfallversorgung/Gutachten Notfallversorgung.pdf.Zugegriffen: 10.Okt. 2017
15. Lapena Lopez de Armentia S, Reguero Celada S, Garcia Rabanal M et al (1996) Estudio epidemiologico de las urgencias pediatricas en un hospital general. Factores implicados en una demanda inadecuada. An Esp Pediatr 44:121-125

16. Mackway-Jones K, Marsden J, Windle J et al (Hrsg) (2011) Ersteinschätzung in der Notaufnahme, 3. Aufl. Huber, Bern

17. Ogilvie S, Hopgood K, Higginson I et al (2016) Why do parents use the emergency department for minor injury and illness? A cross-sectional questionnaire. JRSM Open 7:1-10

18. Porter ME, Guth C (2012) Redefining German health care. Springer, Berlin

19. Redstone P, Vancura JL, Barry D et al (2008) Nonurgent use of the emergency department. J Ambul Care Manage 31:370-376

20. Roukema J, Steyerberg EW, van Meurs A et al (2006) Validity of the Manchester Triage System in paediatric emergency care. Emerg Med J 23:906-910

21. Scherer M, Lühmann D, Kazek A et al (2017) Patienten in Notfallambulanzen. Dtsch Arztebl Int 114:645-652

22. Schlingensiepen I (2017) Notdienst: NRW testet integrierte Leitstelle. ÄrzteZeitung 18.07.2017. https://www.aerztezeitung.de/ politik gesellschaft/berufspolitik/article/940051/ notdienst-nrw-testet-integrierte-leitstelle.html. Zugegriffen:23. Nov. 2017

23. Schmiedhofer M, Möckel M, Slagman A et al (2016) Patient motives behind low-acuity visits to the emergency department in Germany: a qualitative study comparing urban and rural sites. BMJ Open 6:1-11

24. Schöpke T, Plappert T (2011) Kennzahlen von Notaufnahmen in Deutschland. Notfall Rettungsmed 14:371-378

25. Schreyögg J, Bäuml M, Krämer J et al (2014) Forschungsauftrag zur Mengenentwicklung nach § 17b Abs. 9 KHG, Endbericht Juli 2014. Center for Health Economics, Hamburg

26. Schwartau I, Pant H, Borde T et al (2006) Behandlungsdringlichkeit aus ärztlicher und $\mathrm{Pa}$ tientensicht - eine prospektive vergleichende Querschnittsstudie an gynäkologisch-internistischen Notfallambulanzen von drei Berliner Innenstadtkliniken. Geburtshilfe Frauenheilkd 66:263-269

27. Searle J, Muller R, Slagman A et al (2015) Überfüllung der Notaufnahmen. Notfall Rettungsmed 18:306-315

28. Somasundaram R, Geissler A, Leidel BA et al (2016) Beweggründe für die Inanspruchnahme von Notaufnahmen - Ergebnisse einer Patientenbefragung. Gesundheitswesen 17:1-6

29. Stanley R, Zimmerman J, Hashikawa C et al (2007) Appropriateness of children's nonurgent visits to selected Michigan emergency departments. Pediatr Emerg Care 23:532-536

30. Timm NL, Ho ML, Luria JW (2008) Pediatric emergency department overcrowding and impact on patient flow outcomes. Acad Emerg Med 15:832-837

31. van Veen $M$, Steyerberg EW, Ruige $M$ et al (2008) Manchester Triage System in paediatric emergency care: prospective observational study. BMJ 337:1-7
DerAnaesthesist

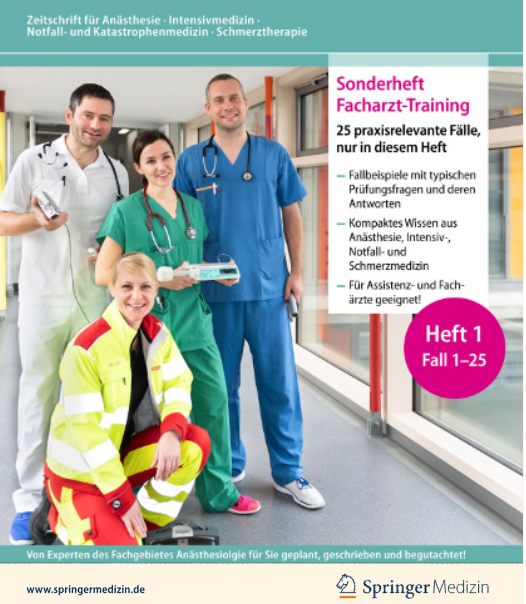

\section{Jetzt neu: Facharzt-Training Anästhesiologie}

Bereiten Sie sich auf die Facharztprüfung vor oder möchten Sie Ihr fachspezisches Wissen mit typischen Fallbeispielen aus der Anästhesiologie aufrischen? Dann ist das Sonderheft von Der Anaesthesist "Facharzt-Training Anästhesiologie" genau das Richtige für Sie.

Sie finden in diesem Heft:

- typische, alltagsnahe Fallbeispiele, systematisch und aktuell aufbereitet

- mit Prüfungsfragen und deren Antworten

- Kompaktes Wissen aus Anästhesie, Intensiv-, Notfall- und Schmerzmedizin

- Von Experten für Sie geplant, geschrieben und begutachtet

Ein zweites Sonderheft, das im November 2019 erscheint, wird die Fallsammlung vervollständigen.

Bestellen Sie „Facharzt-Training Anästhesiologie" (Heft 1) per Mail mit Angabe des Aktionscodes $\mathrm{C} 0017488$ bei: Marie-Luise.Witschel@springer.com 